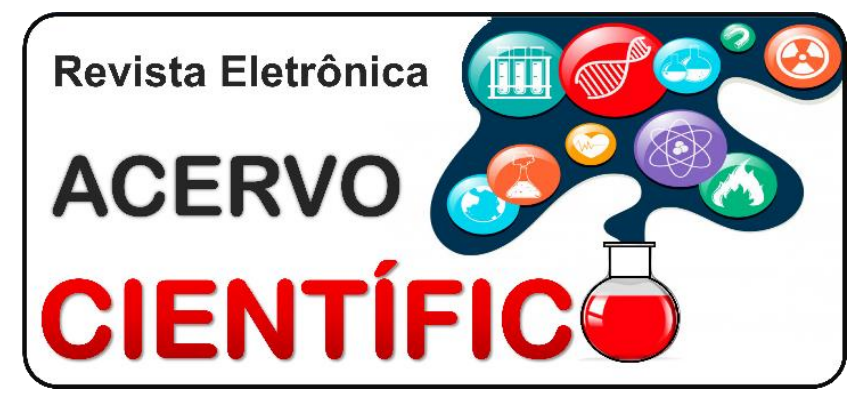

ARTIGO ORIGINAL

Recebido em: 1/2020

Aceito em: 2/2020

Publicado em: 3/2020

\title{
Perfil epidemiológico dos casos de sífilis congênita no Maranhão
}

\author{
Epidemiological profile of congenital syphilis cases in Maranhão
}

Perfil epidemiológico de los casos de sífilis congénita en Maranhão

Thaylana Lysle Silva Lima Leal ${ }^{1 *}$, Elaine da Silva Carneiro, Igor Dias Barrosoㄹ, Taylane Sá Sipaúba ${ }^{1}$, Karen Patrícia Varão de Almeida${ }^{1}$, Laércio Guimarães Leal², Thaynara Sá Sipaúba ${ }^{3}$.

\begin{abstract}
Resumo: O estudo teve como objetivo descrever o perfil epidemiológico dos casos de sífilis congênita no estado do Maranhão no período de 2014 a 2018. Trata-se de um estudo epidemiológico de abordagem quantitativa, de caráter descritivo e exploratório, com coleta de dados realizada na Plataforma do Departamento de Informática do Sistema Único de Saúde (DATASUS). Foi possível observar que o Maranhão teve o maior índice no ano de 2018, correspondendo a 34,6\%. Embora $84,1 \%$ das gestantes tenham realizado o pré-natal e 47,2\% tenham sido diagnosticadas durante a realização do mesmo, 67,8\% 9 realizaram o tratamento de forma inadequada e $12,92 \%$ não o realizaram, esse fato também foi pertinente no que diz respeito ao tratamento do parceiro, onde $54,9 \%$ não o realizaram, colaborando assim para o percentual de $96,7 \%$ casos de sífilis congênita recente. Portanto o estudo evidenciou a alta incidência da SC no Maranhão, e que isto está relacionado a diversos fatores sobretudo ao não tratamento do parceiro e consequentemente a reinfecção da mãe e infecção do feto.
\end{abstract}

Palavras-chave: Sífilis congênita, Gestação, Epidemiologia.

\begin{abstract}
This study aimed to describe the epidemiological profile of cases of congenital syphilis in the state of Maranhão from 2014 to 2018. This is an epidemiological study of quantitative approach, descriptive and exploratory, with data collection performed at the Platform of the Informatics Department of the Unified Health System (DATASUS). It was observed that Maranhão had the highest index in the year 2018, corresponding to $34.6 \%$. Although $84.1 \%$ of pregnant women underwent prenatal care and $47.2 \%$ were diagnosed during prenatal care, $67.8 \% 9$ underwent treatment inappropriately and $12.92 \%$ did not receive it. It was pertinent with regard to the treatment of the partner, where $54.9 \%$ did not perform it, thus contributing to the percentage of $96.7 \%$ cases of recent congenital syphilis. Therefore, the study showed the high incidence of CS in Maranhão, and this is related to several factors, especially the non-treatment of the partner and consequently the mother's reinfection and fetal infection.
\end{abstract}

Key words: Congenital syphilis, Pregnancy, Epidemilogy.

\footnotetext{
1 Universidade Estadual do Maranhão (UEMA), Colinas - MA. *E-mail: thaylanalysle@gmail.com

2 Universidade Federal do Piauí (UFPI), Picos - PI.

${ }^{3}$ Faculdade Pitágoras de Imperatriz (FPI), Imperatriz - MA.
} 
Resumen: El objetivo de este estudio fue describir el perfil epidemiológico de los casos de sífilis congénita en el estado de Maranhão de 2014 a 2018. Este es un estudio epidemiológico descriptivo y exploratorio con un enfoque cuantitativo, con recolección de datos realizada en el Departamento de Informática del Sistema Único de Salud (DATASUS). Se pudo observar que Maranhão tuvo el índice más alto en 2018, correspondiente al $34,6 \%$. Aunque el $84.1 \%$ de las mujeres embarazadas se sometieron a atención prenatal y el $47.2 \%$ fueron diagnosticadas durante la atención prenatal, el $67.8 \% 9$ se sometió a un tratamiento inadecuado y el $12.92 \%$ no lo recibió. Fue pertinente con respecto al tratamiento de la pareja, donde el $54,9 \%$ no lo realizó, contribuyendo así al porcentaje del $96,7 \%$ de casos de sífilis congénita reciente. Por lo tanto, el estudio mostró la alta incidencia de CS en Maranhão, y esto está relacionado con varios factores, especialmente el no tratamiento de la pareja y, en consecuencia, la reinfección de la madre y la infección fetal.

Palabras clave: Sífilis congénita, Embarazo, Epidemiologia.

\section{INTRODUÇÃO}

A sífilis é uma doença infecciosa de caráter sistêmico transmitida por via sexual e vertical, raramente via transfusão sanguínea. É uma patologia que pode ser prevenida porém quando não tratada precocemente, pode evoluir para um quadro crônico com sequelas irreversíveis. Sendo uma doença de notificação obrigatória (ANDRADE ALMB, et al., 2018).

A Sífilis Congênita (SC), é causada pelo Treponema pallidum, sendo uma doença sexualmente transmissível, sendo transmitida por via transplacentária em qualquer momento da gestação, independente do estágio clínico da doença na gestação. Quando não tratada durante a gestação, resulta em considerável proporção de mortes fetais e neonatais precoces, com alta probabilidade de transmissão vertical, principalmente nas fases primária e secundária, aumentando o risco de mortes perinatais (SARACENI V, et al., 2017).

Dessa forma, a SC se configura como um desafio para a saúde pública brasileira, pois apesar de ser doença de fácil prevenção, diagnóstico e tratamento, percebe-se que há aumento da incidência dessa afecção, associada principalmente às desigualdades sociais e à fragilidade na cobertura e assistência do prénatal oferecido a população, principalmente no nível da atenção básica (FRANÇA ISX, et al., 2015; RODRIGUES VRL, et al., 2017).

A SC pode ser classificada em SC precoce e SC tardia. A SC precoce ocorre quando as manifestações clínicas surgem nos dois primeiros anos de vida e a SC tardia ocorre quando as manifestações surgem após o segundo ano de vida. Essa patologia pode provocar consequências graves para o concepto como aborto, óbito fetal e sequelas motoras, cognitivas, neurológicas, visuais e auditivas. Quando a gestante é precocemente diagnosticada e adequadamente tratada, a transmissão vertical é evitável (ANDRADE ALMB, et al., 2018).

De acordo com a Organização Mundial da Saúde (OMS), em 2016, havia mais de meio milhão (aproximadamente $661 \mathrm{mil}$ ) de casos de sífilis congênita no mundo, resultando em mais de 200 mil natimortos e mortes neonatais. Nesse contexto, representa um desafio para a saúde pública brasileira, apesar de ser doença de fácil prevenção, diagnóstico e tratamento, percebe-se que há aumento da ocorrência dessa afecção, fazendo-se responsável por um grande número de complicações perinatais (OMS, 2019).

A sífilis é uma das infecções sexualmente transmissíveis (IST) mais comuns globalmente, com cerca de 6 milhões de novos casos a cada ano. Sendo a sífilis congênita é a segunda principal causa de morte fetal evitável em todo o mundo, precedida apenas pela malária (OMS, 2019).

No Brasil, o Sistema Único de Saúde (SUS) preconiza, por meio das políticas públicas direcionadas as gestantes e aos seus parceiros sexuais, a prevenção da incidência dessa patologia oferendo diagnóstico e tratamento gratuitos para a população. Ainda assim, a incidência de novos casos de SC tem aumentado consideravelmente, ocasionando diversas complicações para os conceptos/recém-nascidos resultante dessa infecção evitável (LAZARINI FM e BARBOSA DA, 2017). 
Nesse sentido, este artigo objetiva descrever o perfil epidemiológico dos casos de sífilis congênita no Maranhão no período de 2014 a 2018, no intuito de revelar a situação da demanda relacionada à doença e assim oferecer suporte para que mais pesquisas voltadas à temática sejam realizadas.

\section{MÉTODOS}

Trata-se de um estudo epidemiológico de abordagem quantitativa, de caráter descritivo e exploratório. Para obtenção da amostra fez-se coleta de dados na Plataforma do Departamento de Informática do Sistema Único de Saúde (DATASUS), com busca dos casos notificados de sífilis congênita no estado do Maranhão no período de 2015 a 2018, utilizando-se as seguintes variáveis: idade da criança, diagnóstico final, faixa etária da mãe, raça ou cor, realização do pré-natal, momento do diagnóstico da sífilis materna, esquema de tratamento materno, parceiro tratado e óbitos de sífilis congênita em menores de um ano.

As seguintes variáveis foram selecionadas e dispostas na forma de tabelas em número absoluto e relativo: sífilis congênita segundo a idade da criança por ano de diagnóstico (< 7 dias, 7 a 27 dias e 28 a 364 dias), diagnóstico final (sífilis congênita recente, tardia, aborto por sífilis, natimorto por sífilis), faixa etária da mãe (10 a 14 anos, 15 a 19 anos, 20 a 29 anos, 30 a 39 anos, 40 anos ou mais), raça ou cor (branca, preta, amarela, parda, indígena e ignorado) realização do pré-natal (sim, não e ignorado) momento do diagnóstico da sífilis materna (durante o pré-natal, no momento do parto/curetagem, após o parto, não realizado, ignorado), esquema de tratamento materno (adequado, inadequado, não realizado, ignorado), parceiro tratado (sim, não e ignorado), óbitos de sífilis congênita em menores de um ano (números de caso).

Através do estudo pode-se investigar a evolução dos casos durante os últimos anos. Os dados coletados foram referentes a todos os casos de sífilis congênita confirmados no Maranhão período esse concernente aos últimos cinco anos com dados disponíveis no sistema. Por meio do Microsoft Excel 2013 foram realizados cálculos de média e desvio padrão do número de casos no período do estudo. Os resultados e informações obtidos foram apresentados por meio de tabelas.

\section{RESULTADOS}

No período analisado, foram notificados 2433 casos de sífilis congênita no Maranhão em menores de um ano, sendo que a maior frequência ocorreu no ano de 2018 representando $34,6 \%(n=842)$ do total de casos, seguido de $2016 \mathrm{com} 18,0 \%$ ( $n=440), 2015 \mathrm{com} 17,7 \%(n=431)$ e $2017 \mathrm{com} 17,5 \%(n=427)$. O menor percentual se deu no ano de 2014 com percentual de 12,0\% $(n=293)$.

$\mathrm{Na}$ distribuição dos casos segundo a idade da criança, foi observado que a maior prevalência nos anos analisados se deu em menores de 7 dias de vida representando o percentual de $92,7 \%(n=2256)$ do total de casos dos menores de um ano. Considerando todos os casos notificados nesse período, inclusive em maiores de um ano, o diagnóstico final mais prevalente foi o de sífilis congênita recente com $96,7 \%(n=2365)$ dos casos. Em relação ao momento do diagnóstico, 47,2\% $(n=1154)$ ocorreu durante o pré-natal e $24,6 \%(n=$ 602) no momento do parto/curetagem, sendo ainda 15,7\% $(n=383)$ diagnosticado após o parto e 10,6\% $(n=$ 258) tiveram esse campo de notificação ignorado (Tabela 1). 
Tabela 1 - Casos confirmados de sífilis congênita segundo idade da criança, diagnóstico final de sífilis e momento do diagnóstico da sífilis materna. Maranhão, Brasil, 2014-2018.

VARIÁVEIS

\begin{tabular}{|c|c|c|c|c|c|c|c|c|c|c|c|c|}
\hline \multirow{2}{*}{ Idade da Criança } & \multicolumn{2}{|c|}{2014} & \multicolumn{2}{|c|}{2015} & \multicolumn{2}{|c|}{2016} & \multicolumn{2}{|c|}{2017} & \multicolumn{2}{|c|}{2018} & \multicolumn{2}{|c|}{ TOTAL } \\
\hline & $\mathrm{n}$ & $\%$ & $\mathbf{n}$ & $\%$ & $\mathbf{N}$ & $\%$ & $\mathbf{n}$ & $\%$ & $\mathbf{n}$ & $\%$ & $\mathbf{n}$ & $\%$ \\
\hline$<7$ dias & 269 & 90,9 & 390 & 89,9 & 410 & 92,8 & 390 & 90,9 & 797 & 94,4 & 2256 & 92,7 \\
\hline 7 a 27 dias & 8 & 2,7 & 12 & 2,8 & 13 & 2,9 & 11 & 2,6 & 23 & 2,7 & 67 & 2,7 \\
\hline 28 a 364 dias & 16 & 5,4 & 29 & 6,7 & 17 & 3,8 & 26 & 6,1 & 22 & 2,6 & 110 & 4,5 \\
\hline \multirow{2}{*}{$\begin{array}{l}\text { Diagnóstico } \\
\text { final }\end{array}$} & \multicolumn{2}{|c|}{2014} & \multicolumn{2}{|c|}{2015} & \multicolumn{2}{|c|}{2016} & \multicolumn{2}{|c|}{2017} & \multicolumn{2}{|c|}{2018} & \multicolumn{2}{|c|}{ TOTAL } \\
\hline & $\mathbf{n}$ & $\%$ & $\mathbf{n}$ & $\%$ & $\mathbf{N}$ & $\%$ & $\mathbf{n}$ & $\%$ & $\mathbf{n}$ & $\%$ & $\mathbf{N}$ & $\%$ \\
\hline $\begin{array}{l}\text { Sífilis congênita } \\
\text { recente }\end{array}$ & 269 & 90,9 & 419 & 96,5 & 432 & 97,7 & 412 & 96,0 & 833 & 98,7 & 2265 & 96,7 \\
\hline $\begin{array}{l}\text { Sífilis congênita } \\
\text { tardia }\end{array}$ & 2 & 0,7 & 2 & 0,5 & 1 & 0,2 & 2 & 0,5 & 0 & 0,0 & 7 & 0,2 \\
\hline Aborto por sífilis & 10 & 3,4 & 2 & 0,5 & 4 & 0,9 & 4 & 0,9 & 2 & 0,2 & 22 & 0,8 \\
\hline $\begin{array}{l}\text { Natimorto por } \\
\text { sífilis }\end{array}$ & 15 & 5,1 & 11 & 2,5 & 5 & 1,1 & 11 & 2,6 & 9 & 1,1 & 51 & 2,0 \\
\hline \multirow{2}{*}{$\begin{array}{c}\text { Momento do } \\
\text { diagnóstico da } \\
\text { sífilis }\end{array}$} & \multicolumn{2}{|c|}{2014} & \multicolumn{2}{|c|}{2015} & \multicolumn{2}{|c|}{2016} & \multicolumn{2}{|c|}{2017} & \multicolumn{2}{|c|}{2018} & \multicolumn{2}{|c|}{ TOTAL } \\
\hline & $\mathbf{n}$ & $\%$ & $\mathbf{n}$ & $\%$ & $\mathbf{N}$ & $\%$ & $\mathbf{n}$ & $\%$ & $\mathbf{n}$ & $\%$ & $\mathbf{N}$ & $\%$ \\
\hline $\begin{array}{c}\text { Durante o pré- } \\
\text { natal }\end{array}$ & 81 & 27,4 & 139 & 32,0 & 198 & 44,8 & 209 & 48,7 & 527 & 48,7 & 1154 & 47,2 \\
\hline $\begin{array}{l}\text { No momento do } \\
\text { parto/curetagem }\end{array}$ & 87 & 29,4 & 126 & 29,0 & 105 & 23,8 & 110 & 25,6 & 174 & 20,6 & 602 & 24,6 \\
\hline Após o parto & 61 & 20,6 & 82 & 18,9 & 99 & 22,4 & 64 & 14,9 & 77 & 9,1 & 383 & 15,7 \\
\hline Não realizado & 6 & 2,0 & 7 & 1,6 & 6 & 1,4 & 15 & 3,5 & 14 & 1,7 & 48 & 2,0 \\
\hline Ignorado & 61 & 20,6 & 80 & 18,4 & 34 & 7,7 & 31 & 7,2 & 52 & 6,2 & 258 & 10,6 \\
\hline
\end{tabular}

Fonte: MS/SVS/DCCI - Departamento de Doenças de Condições Crônicas e Infecções Sexualmente Transmissíveis, 2020. 
Quanto à faixa etária da mãe, a mais predominante foi de 20 a 29 anos com 53,7\% ( $n=1314)$ do total de casos, seguido das faixas etárias de 15 a 19 anos com 23,1\% $(n=565)$ e de 30 a 39 anos com $18,4 \%(n=$ 452). Referente a raça ou cor da mãe, a raça parda foi mais evidente entre as demais, apresentando 0 percentual de $83,4 \%(n=2041)$ do total de casos (Tabela 2).

Tabela 2- Casos confirmados de sífilis congênita segundo faixa etária e raça ou cor da mãe. Maranhão, Brasil, 2014-2018.

\section{VARIÁVEIS}

ANOS ANALISADOS

\begin{tabular}{|c|c|c|c|c|c|c|c|c|c|c|c|c|}
\hline \multirow{2}{*}{ Faixa etária da mãe } & \multicolumn{2}{|c|}{2014} & \multicolumn{2}{|c|}{2015} & \multicolumn{2}{|c|}{2016} & \multicolumn{2}{|c|}{2017} & \multicolumn{2}{|c|}{2018} & \multicolumn{2}{|c|}{ TOTAL } \\
\hline & $\mathrm{n}$ & $\%$ & $\mathbf{n}$ & $\%$ & $\mathbf{N}$ & $\%$ & $\mathbf{n}$ & $\%$ & $\mathbf{n}$ & $\%$ & $\mathbf{n}$ & $\%$ \\
\hline 10 a 14 anos & 5 & 1,7 & 7 & 1,6 & 9 & 2,0 & 8 & 1,9 & 9 & 1,1 & 38 & 1,5 \\
\hline 15 a 19 anos & 87 & 29,4 & 100 & 23,0 & 98 & 22,2 & 100 & 23,3 & 180 & 21,3 & 565 & 23,1 \\
\hline 20 a 29 anos & 145 & 49,0 & 222 & 51,2 & 245 & 55,4 & 225 & 52,4 & 477 & 56,5 & 1314 & 53,7 \\
\hline 30 a 39 anos & 53 & 17,9 & 94 & 21,7 & 71 & 16,1 & 82 & 19,1 & 152 & 18,0 & 452 & 18,4 \\
\hline 40 anos ou mais & 4 & 1,4 & 2 & 0,5 & 6 & 1,4 & 5 & 1,2 & 10 & 1,2 & 27 & 1,1 \\
\hline Ignorado & 2 & 0,7 & 9 & 2,1 & 13 & 2,9 & 9 & 2,1 & 16 & 1,9 & 49 & 2,0 \\
\hline \multirow{2}{*}{ Raça ou cor da mãe } & \multicolumn{2}{|c|}{2014} & \multicolumn{2}{|c|}{2015} & \multicolumn{2}{|c|}{2016} & \multicolumn{2}{|c|}{2017} & \multicolumn{2}{|c|}{2018} & \multicolumn{2}{|c|}{ TOTAL } \\
\hline & $\mathbf{n}$ & $\%$ & $\mathbf{n}$ & $\%$ & $\mathbf{N}$ & $\%$ & $\mathbf{n}$ & $\%$ & $\mathbf{n}$ & $\%$ & $\mathbf{n}$ & $\%$ \\
\hline Branca & 21 & 7,1 & 43 & 9,9 & 25 & 5,7 & 30 & 7,0 & 69 & 8,2 & 188 & 7,6 \\
\hline Preta & 13 & 4,4 & 23 & 5,3 & 32 & 7,2 & 17 & 4,0 & 40 & 4,7 & 125 & 5,1 \\
\hline Amarela & 1 & 03 & 1 & 0,2 & 4 & 0,9 & 0 & 0 & 3 & 0,4 & 9 & 0,3 \\
\hline Parda & 249 & 84,1 & 351 & 80,9 & 370 & 83,7 & 361 & 84,1 & 710 & 84,1 & 2041 & 83,4 \\
\hline Indígena & 2 & 0,7 & 1 & 02 & 5 & 1,1 & 1 & 0,2 & 3 & 0,4 & 12 & 0,4 \\
\hline Ignorada & 10 & 3,4 & 15 & 3,5 & 6 & 1,4 & 20 & 4,7 & 19 & 2,3 & 70 & 2,8 \\
\hline
\end{tabular}

Fonte: MS/SVS/DCCI - Departamento de Doenças de Condições Crônicas e Infecções Sexualmente Transmissíveis, 2020.

No que diz respeito a realização do pré-natal $84,1 \%(n=2056)$ das mães realizaram o pré-natal, porém em relação ao tratamento materno $67,8 \% 9(n=1660)$ o realizaram de forma inadequada, $12,92 \%(n=316)$ não o realizaram e apenas $3,68 \%(n=90)$ realizaram de maneira adequada, o restante $(15,5 \% n=379)$ tiveram esse campo ignorado. Esse fato também foi pertinente no que diz respeito ao tratamento do parceiro, onde $54,9 \%(n=1344)$ não realizaram o tratamento e apenas $16,9 \%(n=414)$ o realizaram. O percentual de ignorados nessa variável também foi considerável com 28,0\% $(n=687)$, indicando falha na notificação dos dados (Tabela 3). Dos casos notificados nesse período 1,7\% $(n=42)$ evoluíram para óbito em menores de um ano. 
Tabela 3- Casos confirmados de sífilis congênita segundo a realização do pré-natal, e tratamento materno e do parceiro. Maranhão, Brasil, 2014-2018.

VARIÁVEIS

\begin{tabular}{|c|c|c|c|c|c|c|c|c|c|c|c|c|}
\hline \multirow{2}{*}{$\begin{array}{l}\text { Realização de } \\
\text { pré-natal }\end{array}$} & \multicolumn{2}{|c|}{2014} & \multicolumn{2}{|c|}{2015} & \multicolumn{2}{|c|}{2016} & \multicolumn{2}{|c|}{2017} & \multicolumn{2}{|c|}{2018} & \multicolumn{2}{|c|}{ TOTAL } \\
\hline & $\mathrm{n}$ & $\%$ & $\mathrm{n}$ & $\%$ & $\mathbf{N}$ & $\%$ & $\mathrm{n}$ & $\%$ & $\mathrm{n}$ & $\%$ & $\mathbf{n}$ & $\%$ \\
\hline Sim & 241 & 84,4 & 345 & 79,5 & 364 & 82,4 & 347 & 80,9 & 759 & 89,9 & 2056 & 84,1 \\
\hline Não & 50 & 16,9 & 61 & 14,1 & 63 & 14,3 & 63 & 14,7 & 67 & 7,9 & 304 & 12,4 \\
\hline Ignorado & 5 & 1,7 & 28 & 6,5 & 15 & 3,4 & 19 & 4,4 & 18 & 2,1 & 85 & 3,5 \\
\hline
\end{tabular}

\begin{tabular}{|c|c|c|c|c|c|c|c|c|c|c|c|c|}
\hline \multirow{2}{*}{$\begin{array}{c}\text { Tratamento } \\
\text { materno }\end{array}$} & \multirow[b]{2}{*}{$\mathbf{n}$} & \multirow[b]{2}{*}{$\%$} & \multirow[b]{2}{*}{$n$} & \multirow[b]{2}{*}{$\%$} & \multirow[b]{2}{*}{$\mathbf{N}$} & \multirow[b]{2}{*}{$\%$} & \multirow[b]{2}{*}{$\mathbf{n}$} & \multirow[b]{2}{*}{$\%$} & \multirow[b]{2}{*}{$n$} & \multirow[b]{2}{*}{$\%$} & \multirow[b]{2}{*}{$\mathbf{n}$} & \multirow[b]{2}{*}{$\%$} \\
\hline & & & & & & & & & & & & \\
\hline Adequado & 4 & 1,4 & 11 & 2,5 & 18 & 4,1 & 23 & 5,4 & 34 & 4,0 & 90 & 3,6 \\
\hline Inadequado & 137 & 46,3 & 231 & 53,2 & 318 & 71,9 & 312 & 72,7 & 662 & 78,4 & 1660 & 67,8 \\
\hline Não realizado & 37 & 12,5 & 65 & 15,0 & 52 & 11,8 & 67 & 15,6 & 95 & 11,3 & 316 & 12,9 \\
\hline Ignorado & 118 & 39,9 & 127 & 29,3 & 54 & 12,2 & 27 & 6,3 & 53 & 6,3 & 379 & 15,5 \\
\hline
\end{tabular}

\begin{tabular}{cccccccccccccc}
$\begin{array}{c}\text { Parceiro } \\
\text { tratado }\end{array}$ & $\mathbf{N}$ & $\%$ & $\mathbf{n}$ & $\%$ & $\mathbf{N}$ & $\%$ & $\mathbf{n}$ & $\%$ & $\mathbf{n}$ & $\%$ & $\mathbf{n}$ & $\%$ \\
\cline { 2 - 12 } & 36 & 1,2 & 52 & 12,0 & 86 & 19,5 & 87 & 20,3 & 153 & 18,1 & 414 & 16,9 \\
\hline Sim & 122 & 41,2 & 227 & 52,3 & 248 & 56,1 & 240 & 55,9 & 507 & 60,1 & 1344 & 54,9 \\
\hline Igna & 138 & 46,6 & 155 & 35,7 & 108 & 24,4 & 102 & 23,8 & 184 & 21,8 & 687 & 28,0
\end{tabular}

Fonte: MS/SVS/DCCI - Departamento de Doenças de Condições Crônicas e Infecções Sexualmente Transmissíveis, 2020.

\section{DISCUSSÃO}

No período estudado, o Maranhão apresentou número de casos de SC correspondente a 2,2\% dos casos registrados no Brasil, sendo que 2018 , o último ano analisado correspondeu a pouco mais de um terço $(34,6 \%)$ dos casos no período de estudo. Assim, observou-se a crescente incidência de novos casos, e isso pode estar atribuído a diversos fatores como a diminuição de número de sub-registros e consequentemente o aumento das notificações, bem como ações de vigilância epidemiológica direcionadas para uma melhor abordagem e identificação dos casos da doença no estado (LIMA VC, et al., 2017). 
Segundo Padovani C, et al. (2019), faz-se necessário investir mais em vigilância epidemiológica, pois este é o primeiro passo para controlar a evolução da sífilis congênita, uma vez que sem a notificação dos casos suspeitos, não há, consequentemente a investigação, nem tratamento adequado seja para a gestante ou para o bebê, aumentando assim os casos de eventos decorrentes da doença.

Segundo o Ministério da Saúde (2019) os dados brasileiros mais atuais de acordo com Boletim Epidemiológico de Sífilis 2019, demonstram não só o insucesso em atingir essa meta, como também as crescentes taxas de incidência da sífilis no Brasil em comparação ao ano de 2016 expressos no Boletim Epidemiológico, foram notificados 87.593 casos de sífilis adquirida, 37.436 casos de sífilis em gestantes e 20.474 casos de sífilis congênita - entre eles, 185 óbitos - no Brasil. Em contrapartida em 2018, foram notificados no Sinan 158.051 casos de sífilis adquirida (taxa de detecção de 75,8 casos/100.000 habitantes); 62.599 casos de sífilis em gestantes (taxa de detecção de 21,4/1.000 nascidos vivos); 26.219 casos de sífilis congênita (taxa de incidência de 9,0/1.000 nascidos vivos); e 241 óbitos por sífilis congênita (taxa de mortalidade de $8,2 / 100.000$ nascidos vivos).

Observa-se, assim, um aumento significativo dessa taxa nos últimos anos, ainda em 2016, foi declarado no Sistema de Informação de Mortalidade (SIM) um total de 185 óbitos por sífilis em crianças menores de um ano, o que corresponde a um coeficiente de mortalidade de 6,1 por 100.000 nascidos vivos. Com relação ao tratamento, em 2016, 88,9\% das prescrições foram de penicilina benzatina (pelo menos uma dose) e 2,1\% foram de outros esquemas. Em $4,7 \%$ dos casos não houve prescrição, e em 4,2\% não havia informação (ignorado). As proporções de prescrição de penicilina na estratificação por UF variaram de 96,1\%, em Sergipe, a 70,5\%, no Maranhão (BRASIL, 2019).

Existe uma tendência de sífilis congênita terem ocorrência mais elevada em municípios maiores, eventualmente em razão de maior velocidade de disseminação em cidades com maior densidade populacional, em um estudo realizado por Guimarães TA, et al. (2018), evidenciou o município com maior números de casos notificados foi a capital, de São Luís, seguido, por Imperatriz, ambos constituem os municípios com maior densidade demográfica do estado, o que pode justificar a maior ocorrência de casos. Sendo assim, esses municípios demandam uma maior atenção referente a adoção de medidas profiláticas para a diminuição dos números de casos nesses estados.

O estudo aponta que maioria dos diagnósticos da SC se deram durante a realização do pré-natal, cerca de $47,2 \%$ ( $n=1154)$, apresentando um aumento progressivo dos números de casos diagnosticados ao longo dos anos, onde o menor índice referente a 2014, foi de apenas $27 \%$ ( $n=81)$ e o maior em $2018 \mathrm{com} \mathrm{48,7 \%}$ $(n=527)$. Diante das altas ocorrências no cenário nacional, o Sistema Único de Saúde (SUS), desenvolveu planos e estratégias, como por exemplo a rede cegonha e a implantação dos testes rápidos para a triagem da sífilis na atenção básica, tais estratégias tiveram grande impacto no diagnóstico e na elevação da taxa de detecção da sífilis em gestante (MASCHIO-LIMA T, et al., 2019).

Embora $84,1 \%(n=2056)$ das gestantes tenham realizado o pré-natal e tenham sido diagnosticadas durante a realização do mesmo, foram notificados 2265 (96,7\%) casos de sífilis congênita recente. Dessa forma, a falta do tratamento do parceiro sexual e da maioria das gestantes com sífilis, pode ter contribuído muito para as possibilidades de reinfecção e falhas na adequabilidade do tratamento das mulheres (CARDOSO ARP, et al., 2018).

O pré-natal tem um papel importante na redução dos desfechos perinatais negativos, ao monitorar o desenvolvimento da gravidez, diagnosticar e tratar intercorrências clínicas e obstétricas com repercussão para a saúde materna e fetal, realiza ações profiláticas específicas e reduzir a exposição da gestante e do feto a fatores de risco. Vale ressaltar que as ações preventivas da SC, estão ligadas diretamente aos cuidados com a gestante durantes as consultas de pré-natal, com a realização dos testes sorológicos para sífilis durante a gestação, tratamento preciso e oportuno da infecção diagnosticada, instituída o mais precoce, tanto para a mulher como para o parceiro (MARASCHIN MS, et al., 2019). Apesar do MS orientar a realização de testes diagnósticos dessa patologia, a alta taxa de incidência da SC pode estar associada a não realização desses testes e ainda a condutas impróprias como o não-comparecimento do parceiro ao tratamento. A SC pode decorrer por inúmeras razões como o acesso tardio para evitar um evento adverso por parte das mulheres, a 
não solicitação do teste treponêmico, ou mesmo aquelas mulheres que foram tratadas podem ter sido reinfectadas pelos parceiros sexuais não tratados (FAVERO MLDC, et al., 2019).

Um estudo realizado por Rodrigues VLR, et al. (2017) evidenciaram que um dos maiores problemas no tratamento da sífilis também está nos antibióticos de segunda escolha preconizados e disponíveis no SUS do Brasil (Doxiciclina e Ceftriaxona) os mesmos possuem uma posologia recomendada a um período de tempo entre 8 e 15 dias, o que prejudica ainda mais a adesão ao esquema terapêutico completo, resultando em elevadas chances de desenvolvimento de resistência do Treponema pallidum. O que pode resultar, ainda, em falhas no tratamento e consequentes reinfecções nos casos em que as gestantes são corretamente tratadas, mas seus parceiros não.

Nesse sentido, os dados obtidos na pesquisa evidenciaram que o percentual do parceiro não tratado foi de em $54,9 \%(n=1344)$, e em $28,0 \%(n=687)$ das notificações não foi informado a realização do tratamento, o que remete a um acréscimo no número de parceiros não tratados. Além disso, o índice de tratamento materno inadequado foi de $67,8 \%(n=1660)$ e $28,4 \%(n=695)$ não realizaram ou teve esse campo ignorado durante a notificação, apontando um percentual de apenas 3,6\% $(n=90)$ de tratamento adequado. Esse achado pode estar relacionado também às deficiências que permanecem na atenção primária do país, principalmente, no momento do pré-natal. Apesar dos resultados indicarem um aumento considerável na adesão às consultas de pré-natal e, consequentemente, um diagnóstico mais precoce da infecção, 0 tratamento das gestantes ainda é majoritariamente inadequado e seus parceiros, em sua maioria, não são tratados, contribuindo assim para desfechos indesejáveis (FRANÇA ISX, et al., 2015).

De acordo com a OMS, deve-se englobar o conhecimento pelos países dos três indicadores mais relevantes relacionados a transmissão vertical para a monitorização e eliminação da SC. Os três principais indicadores são: a proporção de gestantes em acompanhamento pré-natal testadas para sífilis, a proporção de gestantes soropositivas para SG e a proporção de gestantes com diagnóstico de sífilis tratadas adequadamente (OMS, 2011).

No intuito de colaborar para a mudança no quadro epidemiológico da sífilis congênita e alcançar o objetivo de controle dessa infecção, é necessário que haja um esforço mútuo com assistência pré-natal de qualidade, exames laboratoriais em tempo hábil, tratamento do casal e conscientização de todos os envolvidos. Desse modo, atuação da Atenção Básica é essencial nesse processo por meio da Estratégia de Saúde da Família, visto que é uma das principais portas de entrada para os serviços de saúde estando mais próxima da população, principalmente por meio dos agentes comunitários de saúde para busca ativa às gestantes (LAFETÁ KRG, et al., 2016).

\section{CONCLUSÃO}

Os achados apresentados no presente estudo evidenciam que, dentro do período em análise, taxas médias de incidência da SC no Maranhão ficaram acima do parâmetro nacional estabelecido e as características analisadas dos casos apontam para uma problemática relacionada a diversos fatores sobretudo ao não tratamento do parceiro e consequentemente a reinfecção da mãe e infecção do feto o que mostra que ainda existe uma baixa efetividade da assistência ao pré-natal. Assim sendo, a atenção primária deve-se atentar também ao comparecimento do parceiro no pré-natal para realização dos testes rápidos e, se necessário, tratamento precoce. Para isso a Atenção Básica através da Estratégia Saúde da Família deve instituir novas medidas de abordagem na assistência pré-natal e que as Secretarias Municipais criem ações de controle mais eficazes, onde cada caso novo deve ser interpretado como uma falha no processo construído para a contenção da transmissão vertical e horizontal da doença.

\section{REFERÊNCIAS}

1. ANDRADE ALMB, et al. Diagnóstico tardio de sífilis congênita: uma realidade na atenção à saúde da mulher e da criança no brasil. Revista Paulista de Pediatria, São Paulo, 2018; 36(3): 376-381. 
2. BRASIL. Ministério da Saúde. Sífilis. Boletim epidemiológico, Brasilia, 2019.

3. CARDOSO ARP, et al. Análise dos casos de sífilis gestacional e congênita nos anos de 2008 a 2010 em Fortaleza, Ceará, Brasil. Ciência \& Saúde Coletiva, Ceará, 2018; 23(2): 563-574.

4. FAVERO MLDC, et al. Sífilis congênita e gestacional: notificação e assistência pré-natal. Arch. Health. Sci, Maringá, $2019 ; 26(1): 2-8$.

5. FRANÇA ISX, et al. Fatores associados à notificação da sífilis congênita: um indicador de qualidade da assistência pré-natal. Revista da Rede de Enfermagem do Nordeste, Ceará, 2015; 16(3): 374-381.

6. LAFETÁ KRG et al. Sífilis materna e congênita, subnotificação e difícil controle. Rev Bras Epidemiol, 2016; 15(1): 63-74.

7. LAZARIN FM, BARBOSA DA. Intervenção educacional na Atenção Básica para prevenção da sífilis congênita. Rev. Latino-am. Enfermagem, São Paulo, 2017; 25(e2845): 2-9.

8. GUIMARÃES TA, et al. Sífilis em gestantes e sífilis congênita no Maranhão. Arq. Ciênc. Saúd, Maranhão, 2018; 25(2): 24-30.

9. LIMA VC, et al. Perfil epidemiológico dos casos de sífilis congênita em um município de médio porte no nordeste brasileiro. J. Health Biol Sci, 2017; 5(1): 56-61.

10. MARASCHIN MS, et al. Sífilis materna e sífilis congênita notificados em um hospital de ensino. Revista Nursing, 2019; 22(257): 3209-3213.

11. MASCHIO-LIMA T, et al. Perfil epidemiológico de pacientes com sífilis congênita e gestacional em um município do Estado de São Paulo, Brasil. Revista Brasileira de Saúde Materno Infantil, [s.I.], 2019; 19(4): 865-872.

12. OMS, Organização Mundial da Saúde. Métodos para vigilância e monitoramento da eliminação da sífilis congênita nos sistemas existentes. 2011.

13. OMS, Organização Mundial da Saúde. Organização Mundial da Saúde pública novas estimativas sobre sífilis congênita. 2019.

14. PADOVANI C, et al. Sífilis na gestação: associação das características maternas e perinatais em região do sul do Brasil. Rev. Latino-am. Enfermagem, Maringá, 2018; 26(e3019): 1-10.

15. RODRIGUES VLR, et al. Sífilis Congênita na Perspectiva de um Desafio para a Saúde Pública. Universidade Tiradentes, 2017; 9(12): 1-4.

16. SARACENI V, et al. Vigilância epidemiológica da transmissão vertical da sífilis: dados de seis unidades federativas no Brasil. Revista Panam Salud Publica, 2017; 41(44): 1-8. 